\title{
Association of pulse pressure, pulse pressure index, and ambulatory arterial stiffness index with kidney function in a cross-sectional pediatric chronic kidney disease cohort from the CKiD study
}

\author{
Rupesh Raina MD ${ }^{1,2}$ (D) | Shyam Polaconda BS ${ }^{3}$ | Nikhil Nair BS ${ }^{4}$ | \\ Ronith Chakraborty BS ${ }^{2}$ (D) | Sidharth Sethi MD ${ }^{5}$ (D) | Vinod Krishnappa MD ${ }^{6}$ (D) | \\ Gaurav Kapur MD ${ }^{7}$ | Maroun Mhanna MD $^{8}$ | Kirsten Kusumi MD ${ }^{1}$ (D)
}

${ }^{1}$ Department of Pediatric Nephrology, Akron Children's Hospital, Akron, Ohio

${ }^{2}$ Akron Nephrology Associates, Cleveland Clinic Akron General, Akron, Ohio

${ }^{3}$ Department of Biomedical Engineering, Case Western Reserve University,

Cleveland, Ohio

${ }^{4}$ Department of Chemistry, Case Western Reserve University, Cleveland, Ohio

${ }^{5}$ Department of Pediatric Nephrology, Medanta, The Medicity, Gurgaon, India

${ }^{6}$ Consortium of Eastern Ohio Master of Public Health student, Northeast Ohio Medical University, Rootstown, Ohio

${ }^{7}$ Division of Pediatric Nephrology and Hypertension, Carman and Ann Adams Department of Pediatrics, Children's Hospital of Michigan, Wayne State University, Detroit, Michigan

${ }^{8}$ Department of Pediatrics, MetroHealth, Cleveland, Ohio

\section{Correspondence}

Rupesh Raina, MD, FAAP, FACP, FASN, FNKF, Consultant Nephrologist, AdultPediatric Kidney Disease/Hypertension, Department of Nephrology, Cleveland Clinic Akron General and Akron Children's Hospital, Akron, OH, USA.

Emails: rraina@akronchildrens.org; raina@ akronnephrology.com

\begin{abstract}
The morbidity and mortality of adult and pediatric chronic kidney disease (CKD) and end-stage renal disease (ESRD) populations are mainly driven by cardiovascular disease (CVD). Improving CVD outcomes focuses on risk assessment of factors including diastolic blood pressure (DBP), systolic blood pressure (SBP), left ventricular mass index (LVMI), pulse pressure (PP), and pulse pressure index (PPi), which is calculated as $\mathrm{PP} / \mathrm{SBP}$. These markers are also proven predictors of CKD progression; however, their role in children has not been established. This study aims to evaluate the relationship between PP, PPi, ambulatory arterial stiffness index (AASI), and proteinuria with kidney function in pediatric CKD patients; it is a retrospective analysis of 620 patients (1-16 years) from the NIDDK Chronic Kidney Disease in Children (CKiD) registry. The authors analyzed data for three separate cohorts: an overall CKD as well as immunological versus non-immunological cause for CKD groups. An inverse relationship was found between SBP, DBP, and PP with iGFR and LVMI in the overall CKD group. Our immunological CKD subgroup showed significantly higher serum creatinine, SBP, DBP, and PP values with significantly lower serum albumin levels compared to the non-immunological group. There were no significant differences with iohexol-based glomerular filtration rate (iGFR), LVMI, PPi, or high-sensitivity C-reactive protein (hsCRP) between the two groups. A subgroup analysis demonstrated that SBP, DBP, and PP all correlated significantly with LVMI in the immunological CKD patients but not the non-immunological subgroup. Additionally, AASI data in the overall CKD population were significantly correlated with PP, PPi, and DBP. This study is one of the first to correlate noninvasive measurements of vascular compliance including PP, PPi, and AASI with iGFR and LVMI in a pediatric CKD cohort. Improving our understanding of surrogate markers for early CVD is integral to improving the care of pediatric CKD population as these patients have yet to develop the hard end points of ESRD, heart failure, myocardial infarction, or stroke.
\end{abstract}




\section{1 | INTRODUCTION}

Chronic kidney disease (CKD) and end-stage renal disease (ESRD) are a significant burden for patients due to their high morbidity and associated mortality; furthermore, CKD and ESRD care is complex and requires a significant portion of our health care resources. ${ }^{1}$ The morbidity and mortality of adult and pediatric CKD/ESRD populations are mainly driven by cardiovascular disease (CVD). ${ }^{2}$ Hypertension (HTN) has long been identified as a paramount independent risk factor for CVD development as well as CKD progression; improving CVD outcomes has focused on risk assessment including analysis of systolic blood pressure (SBP) and diastolic blood pressure (DBP). ${ }^{3}$ However, as medical management of HTN has improved, the onus of care has shifted to prevention including early detection of evolving vascular pathology prior to changes in blood pressure, and recently, the emphasis has shifted to noninvasive arterial elasticity measurements.

The strong prognostic value of arterial stiffness measurements has been firmly established over the last two decades in the adult CKD and ESRD populations. ${ }^{2}$ Furthermore, the authors know that arterial stiffness measurements offer additive predictive value to traditional risk factors with respect to cardiovascular outcomes. ${ }^{2}$ However, measurements of arterial stiffness have not been well evaluated in the pediatric CKD populations. While well-established markers such as pulse wave velocity (PWV) and the augmentation index have excellent prognostic value in adults, they are cumbersome due to the need for specialized equipment and remain mostly utilized in research. ${ }^{4,5}$ Thus, clinicians need arterial stiffness parameters that are accessible for routine practice; two such potential parameters include pulse pressure (PP) and ambulatory arterial stiffness index (AASI). PP is well established and easily calculated as the difference between maximal systolic blood pressure (SBP) and minimal diastolic blood pressure (DBP), and increased PP is associated with poor CKD outcomes in adults. ${ }^{6}$ AASI is a newer method and is mathematically derived from 24-hour ambulatory blood pressure monitoring (ABPM). ${ }^{7}$ The utility of PP has its limitations, however including its propensity to increase or decrease in an individual while not reflecting absolute blood pressure levels. ${ }^{8}$ To account for this, Peng-Lin and Yue-Chun proposed a new parameter: pulse pressure index (PPi) (calculated as (SBP - DBP)/SBP), as a more accurate indirect measure of vascular compliance. ${ }^{6}$ Its structure is similar to the fluid-flow analog presented by Ohm's law (pressure gradient $=$ volume flow rate $\times$ resistance). ${ }^{8}$ Thus, PPi accounts for absolute blood pressure changes and is superior to PP alone as an indication of vascular compliance and may potentially be a better predictor of $\mathrm{CV}$ outcomes. 8

Persistent low-grade inflammation is a hallmark of CKD, and increased inflammation is a risk factor for CKD morbidity and mortality in adults. ${ }^{9-12}$ Various immunological biomarkers, including interleukin-1 (IL-1), IL-1 receptor antagonist (IL-1RA), interleukin-6 (IL-6), tumor necrosis factor-alpha (TNF- $\alpha$ ), tumor necrosis factor-beta (TNF- $\beta$ ), high-sensitivity C-reactive protein (hs-CRP), fibrinogen, and serum albumin, have been implicated in CKD progression. ${ }^{12-14}$ However, their role in CKD in children has not been established.

The value of establishing noninvasive prognostic markers for CVD morbidity/mortality as well as loss of kidney function is integral to improving the care of pediatric CKD population as these patients have yet to develop the hard end points of ESRD, heart failure, myocardial infarction, or stroke. Children are thus a population with the greatest potential for preventative care and improvement in long-term outcomes and are deserving of increased research. This study aims to investigate the relationship of PP, PPi, AASI, and proteinuria with kidney function using the Chronic Kidney Disease in Children (CKiD) database from the National Institute of Diabetes and Digestive Kidney Diseases (NIDDK) registry.

\section{2 | MATERIAL AND METHODS}

\section{1 | Study design}

This is a retrospective and a cross-sectional study of 620 pediatric CKD patients (age 1-16 years) from the CKiD database of the NIDDK registry. The correlation analysis of multiple variables present in CKD was retrospectively assessed while the correlation analysis of AASI was cross-sectional. Data in this manuscript were collected by the Chronic Kidney Disease in Children (CKiD) study with clinical coordinating centers (Principal Investigators) at Children's Mercy Hospital at the University of Missouri-Kansas City (Bradley Warady, MD), Children's Hospital of Philadelphia (Susan Furth, MD, PhD), Central Biochemistry Laboratory (George Schwartz, MD) at the University of Rochester Medical Center, and the Data Coordinating Center (Alvaro Muñoz, PhD and Derek Ng, PhD) at Johns Hopkins' Bloomberg School of Public Health. These data include renal function, measurements of $\mathrm{CV}$ risk factors, co-morbidities, neurocognitive functions, and clinical events related to end-stage renal disease (ESRD) and death. Children <1-year-old were excluded from the registry as glomerular filtration rate (GFR) increases during the first year of life, even in the presence of renal insufficiency.

\section{2 | Study population}

Only patients aged 1-16 years with iohexol-based glomerular filtration rate (iGFR) $<60 \mathrm{~mL} / \mathrm{min} / 1.73 \mathrm{~m}^{2}(\mathrm{n}=620)$ were selected for pulse pressure and AASI correlation analysis.

\section{3 | Data collection}

Data regarding demographics (age, sex, race), etiology of CKD, estimated GFR (eGFR) (Schwartz formula), and use of angiotensin-converting enzyme inhibitors/angiotensin receptor blockers (ACEi/ARB) were recorded. Laboratory markers including serum creatinine $(\mathrm{SCr})$, urine protein levels, and immunological markers (high-sensitive 
C-reactive protein (hs-CRP) and serum albumin) data were also collected.

Blood pressure was measured via auscultation using an aneroid sphygmomanometer (Mabis Medic-Kit 5, Mabis Healthcare), with the average of three auscultatory BP measurements utilized for analysis; the averaged HR from these three BP measurements was also used. PP was calculated by subtracting DBP from SBP and PPi was calculated by using the formula PP/SBP. The 24-hour ambulatory blood pressure monitoring (ABPM) was completed utilizing a Spacelabs 90217 monitor (SpaceLabs Healthcare); BP was measured every 20 minutes during the day and night. After completion of the monitoring period, the monitors were processed and analyzed at the ABPM center (University of Texas Health Science Center at Houston). Requirements for analysis included that the monitor was worn for $\geq 21$ hours and $\geq 18$ hours with $\geq 1$ successful BP measurement per hour. ${ }^{15}$ The AASI was calculated from unedited recordings as follows: The regression of diastolic on systolic BP was estimated for each participant (not forcing the regression line through 0 ) and AASI $=(1$ - regression slope $) .{ }^{4}$ Echocardiograms by $\mathrm{M}$-mode and Doppler were performed at individual participating centers, with the official read and analyses performed by the Cardiovascular Core Imaging Research Laboratory at Cincinnati Children's Hospital Medical Center, to measure left ventricular mass (LVM). ${ }^{16}$ Left ventricular mass index (LVMI) was then calculated by indexing the LVM to the height of the participant. ${ }^{17}$ ABPM, AASI, and LVMI measurements were analyzed with BP and laboratory data from the most proximate clinic visit. Correlation analysis was also performed for proteinuria with SBP and DBP. For analysis, significant proteinuria was defined as a calculated urine protein to creatinine ratio $(\mathrm{Up} / \mathrm{c})$ of $0.2<2$, while nephrotic-range proteinuria was defined as a calculated Up/c $\geq 2.0$. $^{18}$

\section{4 | Statistical analysis}

Descriptive statistics (mean, standard deviation, and percentage) were used to describe study population demographics, measures of renal function, blood pressure variables, and etiology of CKD. The correlation of multiple variables within the overall CKD group as well as between the immunological and non-immunological CKD groups were evaluated utilizing the Pearson's correlation and backward logistic regression analysis to adjust for any potential risk factors. $t$ tests were utilized to check for significant differences in renal function measures, blood pressure variables, immunological biomarkers, and use of ACEi/ARB between immunological and non-immunological CKD groups. Specifically, for proteinuria analysis, linear regression was performed and compared to the normal range Up/c $(<0.2)$. For AASI calculations, the Fisher exact test was used to test differences between proportions while the t tests were performed to compare the continuous data across the various CKD groups. Any variables of interest with a highly skewed distribution were log-transformed before analysis. Additionally, univariate logistic regression analysis was used to calculate the significance F-factors while the independently
TABLE 1 Demographics and clinical characteristics at baseline

\begin{tabular}{|ll|}
\hline Characteristics & $\begin{array}{l}\text { Mean } \pm \text { SD } \\
(n=620)\end{array}$ \\
\hline Age $(y)$ & $9.63 \pm 4.34$ \\
\hline Sex (male $(\%))$ & 59.7 \\
\hline Weight $(\mathrm{kg})$ & $40.4 \pm 22.5$ \\
\hline Serum creatinine $(\mu \mathrm{mol} / \mathrm{L})$ & $50 \pm 31$ \\
\hline iGFR $\left(\mathrm{mL} / \mathrm{min} / 1.73 \mathrm{~m}^{2}\right)$ & $37.9 \pm 12.1$ \\
\hline LVMI & $30.9 \pm 9.3$ \\
\hline SBP (mm Hg) & $107.9 \pm 13.5$ \\
\hline DBP (mm Hg) & $66.3 \pm 11.4$ \\
\hline PP (mm Hg) & $41.7 \pm 10.8$ \\
\hline PPi & $0.38 \pm 0.8$ \\
\hline ACEi/ARB (\%) & $50.5(n=313)$ \\
\hline Race & \\
\hline Caucasian & $69.03 \%(n=428)$ \\
\hline African American (AA) & $15.16 \%(n=94)$ \\
Asian & $2.26 \%(n=14)$ \\
American Indian & $1.13 \%(n=7)$ \\
Native Hawaiian & $0.48 \%(n=3)$ \\
Other & $4.03 \%(n=25)$ \\
\hline More than one race (excluding AA) & $4.52 \%(n=28)$ \\
\hline More than one race (including AA) & $3.39 \%(n=21)$ \\
\hline
\end{tabular}

Abbreviations: ACEi, angiotensin-converting enzyme inhibitor; ARB, angiotensin receptor blocker; DBP, diastolic blood pressure; eGFR, estimated glomerular filtration rate; iGFR, iohexol-based GFR; LVMI, left ventricular mass index; PP, pulse pressure; PPi, pulse pressure index; SBP, systolic blood pressure.

associated variables with each stiffness parameter were evaluated by multiple linear regression using an ordinary least squares model.

\section{RESULTS}

Patient demographics and baseline characteristics are shown in Table 1. On average (mean \pm standard deviation), patients were $9.63 \pm 4.34$ years old and weighed $40.4 \pm 22.5 \mathrm{~kg}$. The majority of patients were male and Caucasian (59.7\% and 69.03\%, respectively). Other characteristics of the studied population are shown in Table 1. The most common causes of CKD were obstructive uropathies followed by aplastic/hypoplastic/dysplastic kidneys and reflux nephropathy (Table 2).

Among all patients ( $n=620), 16 \%(99 / 620)$ had CKD due to immunological causes while 64\% $(521 / 620)$ had CKD due to non-immunological causes (Table 2). In comparison to the non-immunological group, patients with immunological CKD had significantly higher SCr levels, SBP, DBP, and PP (Table 3). Serum albumin was significantly lower in the immunological than the non-immunological CKD group (Table 3). In contrast, there were no significant differences in iGFR, LVMI, PPi, or hs-CRP between the two 
TABLE 2 Causes of chronic kidney disease

\begin{tabular}{|c|c|}
\hline Immunological diseases & $\begin{array}{l}\% \text { of patients } \\
(n=99)\end{array}$ \\
\hline Hemolytic uremic syndrome & $5 \%(n=31)$ \\
\hline Systemic immunological disease (including SLE) & $2.74 \%(n=17)$ \\
\hline Pyelonephritis/interstitial nephritis & $1.94 \%(n=12)$ \\
\hline Chronic glomerulonephritis & $1.61 \%(n=10)$ \\
\hline IgA nephropathy (Berger's) & $1.29 \%(n=8)$ \\
\hline Membranoproliferative glomerulonephritis type I & $0.81 \%(n=5)$ \\
\hline Idiopathic crescentic glomerulonephritis & $0.65 \%(n=4)$ \\
\hline Membranous nephropathy & $0.48 \%(n=3)$ \\
\hline Henoch-Schönlein nephritis & $0.48 \%(n=3)$ \\
\hline $\begin{array}{l}\text { Membranoproliferative glomerulonephritis type } \\
\text { II }\end{array}$ & $0.32 \%(n=2)$ \\
\hline Other glomerular diagnosis & $0.65 \%(n=4)$ \\
\hline Non-immunological diseases & $\begin{array}{l}\% \text { of patients } \\
(n=521)\end{array}$ \\
\hline Obstructive uropathy & $17.74 \%(n=110)$ \\
\hline Aplastic/hypoplastic/dysplastic kidneys & $17.74 \%(n=110)$ \\
\hline Reflux nephropathy & $13.71 \%(n=85)$ \\
\hline Focal segmental glomerulosclerosis & $8.39 \%(n=52)$ \\
\hline Polycystic kidney disease (Autosomal recessive) & $3.55 \%(n=22)$ \\
\hline Renal infarct & $3.06 \%(n=19)$ \\
\hline Cystinosis & $1.77 \%(n=11)$ \\
\hline Syndrome of agenesis of abdominal musculature & $1.77 \%(n=11)$ \\
\hline $\begin{array}{l}\text { Medullary cystic disease/juvenile } \\
\text { nephronophthisis }\end{array}$ & $1.61 \%(n=10)$ \\
\hline Familial nephritis (Alport's) & $1.45 \%(n=9)$ \\
\hline Methylmalonic acidemia & $0.97 \%(n=6)$ \\
\hline $\begin{array}{l}\text { Congenital urologic disease (bilateral } \\
\text { hydronephrosis) }\end{array}$ & $0.81 \%(n=5)$ \\
\hline Perinatal asphyxia & $0.65 \%(n=4)$ \\
\hline Congenital nephrotic syndrome & $0.48 \%(n=3)$ \\
\hline Wilms' tumor & $0.48 \%(n=3)$ \\
\hline Polycystic kidney disease (Autosomal dominant) & $0.48 \%(n=3)$ \\
\hline Sickle cell nephropathy & $0.16 \%(n=1)$ \\
\hline Oxalosis & $0.16 \%(n=1)$ \\
\hline Branchio-oto-renal disease/syndrome & $0.16 \%(n=1)$ \\
\hline Other non-glomerular diagnosis & $8.87 \%(n=55)$ \\
\hline
\end{tabular}

Abbreviations: I, immunological; IgA, immunoglobin A; N, non-immunological.

groups. However, there was increased usage of ACEi/ARBs noted in the immunological CKD group compared to the non-immunological CKD group (Table 3).

The correlation of multiple variables was investigated for the overall CKD group (Table 4 and Figure 1). There was a significant correlation between SBP and iGFR $\left(R^{2}=.033 ; P<.001\right)$, SBP and $\mathrm{SCr}$ $\left(R^{2}=.136 ; P<.001\right)$, and SBP and LVMI $\left(R^{2}=.013 ; P<.001\right)$. Similarly, DBP and iGFR $\left(R^{2}=.013 ; P<.001\right), \mathrm{DBP}$ and $\operatorname{SCr}\left(R^{2}=.061 ; P<.001\right)$, and DBP and LVMI $\left(R^{2}=.005 ; P=.035\right)$ were significant. In addition, $\mathrm{PP}$ and $\mathrm{SCr}\left(R^{2}=.039 ; \mathrm{P}<.001\right)$, PP and LVMI $\left(R^{2}=.005 ; P=.029\right)$, and PP and iGFR $\left(R^{2}=.017 ; P=.011\right)$ were significant. Finally, PPi and $\mathrm{SCr}\left(R^{2}=.0005 ; P=.265\right)$ were significant but not PPi and LVMI $\left(R^{2}=.001 ; P=.437\right)$ nor $P P i$ and iGFR $\left(R^{2}=.001 ; P=.309\right)$. There was no significant correlation between proteinuria and SBP $(P=.11)$, nor proteinuria and $\operatorname{DBP}(P=.38$; Table 5$)$. Nephrotic-range proteinuria was also not significantly correlated with SBP $(P=.06)$. However, a significant correlation was found between nephrotic-range proteinuria and DBP $(P=.03)$.

Correlations were investigated for the immunological CKD group (Table 4 and Figure 2). Significant correlations were seen between SBP and iGFR $\left(R^{2}=.058 ; P<.001\right)$, SBP and $\mathrm{SCr}\left(R^{2}=.150 ; P<.001\right)$, and SBP and LVMI $\left(R^{2}=.104 ; P<.001\right)$. Similarly, DBP and iGFR $\left(R^{2}=.070 ; P<.001\right), \mathrm{DBP}$ and $\mathrm{SCr}\left(R^{2}=.103 ; P<.001\right)$, and DBP and LVMI $\left(R^{2}=.04 ; P=.006\right)$ showed significance. In addition, $\mathrm{PP}$ and $\mathrm{SCr}$ $\left(R^{2}=.021 ; P=.001\right)$, and PP and LVMI $\left(R^{2}=.031 ; P=.016\right)$ showed significance. However, there was no correlation between PP and iGFR $\left(R^{2}=.0003 ; P=.755\right)$. Lastly, PPi and $\mathrm{SCr}\left(R^{2}=.009 ; P=.033\right)$ and PPi and iGFR $\left(R^{2}=.021 ; P=.011\right)$ showed significance. However, there was no correlation between PPi and LVMI $\left(R^{2}=.003 ; P=.492\right)$. To adjust for potential risk factors, a backward logistic regression analysis was conducted with LVMI as the dependent variable, and DBP, PP, and serum albumin as independent variables (Table 6). DBP, PP and serum albumin were all significantly associated with LVMI with an odds ratio ( $95 \%$ Confidence Interval; $p$ value) of $0.16(0.05$, $0.28 ; P=.005), 0.20$ (0.07, 0.32; $P=.002)$, and -4.84 (-7.89, -1.78; $P=.002)$, respectively. Logistic regression analysis with iGFR as the dependent variable, and DBP, PP, and serum albumin as independent variables was also performed. DBP was the only statistically significant factor associated with iGFR with an OR of $-0.27(-0.38$, -0.16 ; $P<.001$ ). Logistic regression analysis with $\mathrm{SCr}$ as the dependent variable, and DBP, PP, and serum albumin as independent variables was done. For $\mathrm{SCr}$, both $\mathrm{DBP}$ and $\mathrm{PP}$ were statistically significant with an OR of $0.03(0.03,0.04 ; P<.001)$, and $0.02(0.01$, $0.03 ; P<.001)$, respectively.

Correlations were also investigated for the non-immunological CKD group (Table 4 and Figure 3). There were again significant correlations between SBP and iGFR $\left(R^{2}=.026\right.$; $\left.P<.001\right)$, and SBP and $\mathrm{SCr}\left(R^{2}=.130 ; P<.001\right)$. However, SBP and LVMI $\left(R^{2}=.003\right.$; $P=.156)$ were not significant. Similarly, DBP and iGFR $\left(R^{2}=.004\right.$; $P=.03)$ and $\mathrm{DBP}$ and $\mathrm{SCr}\left(R^{2}=.049 ; P<.001\right)$ showed significance, however DBP and LVMI $\left(R^{2}=.001 ; P=.512\right)$ were not. In addition, $\mathrm{PP}$ and $\mathrm{SCr}\left(R^{2}=.044 ; P<.001\right)$ and $\mathrm{PP}$ and iGFR $\left(R^{2}=.017 ; P<.001\right)$ were significant. However, PP and $\operatorname{LVMI}\left(R^{2}=.001 ; P=.302\right)$ was not. Finally, PPi and $\mathrm{SCr}\left(R^{2}=.002 ; P=.065\right)$ and PPi and iGFR $\left(R^{2}=.005 ; P=.018\right)$ showed significance. However, PPi and LVMI $\left(R^{2}=.001 ; P=.442\right)$ were not significant. To adjust for potential risk factors, a backward logistic regression analysis was conducted with LVMI as the dependent variables and DBP, PP, and serum albumin as independent variables (Table 6). Serum albumin was the only statistically significant factor associated with LVMI with an OR of -2.63 $(-4.41,-0.86 ; P=.004)$. Logistic regression analysis with iGFR as 
TABLE 3 Comparison of clinical parameters between patients of CKD due to immunological and non-immunological diseases

\begin{tabular}{|c|c|c|c|}
\hline Baseline parameters & $\begin{array}{l}\text { CKD due to immunological diseases } \\
(n=99)\end{array}$ & $\begin{array}{l}\text { CKD due to non-immunological diseases } \\
(n=521)\end{array}$ & $P$ \\
\hline Serum creatinine $(\mathrm{mg} / \mathrm{dL})$ & $1.60 \pm 0.70$ & $1.40 \pm 0.67$ & .0069 \\
\hline LVMI & $31.59 \pm 9.59(n=51)$ & $32.91 \pm 9.65(n=317)$ & .3648 \\
\hline $\mathrm{SBP}(\mathrm{mm} \mathrm{Hg})$ & $112.97 \pm 12.73$ & $105.30 \pm 12.85$ & $<.0001$ \\
\hline $\mathrm{PP}(\mathrm{mm} \mathrm{Hg})$ & $44.07 \pm 10.88$ & $40.12 \pm 10.55$ & .0007 \\
\hline PPi & $0.39 \pm 0.8$ & $0.38 \pm 0.8$ & .9046 \\
\hline ACEi/ARB (\%) & 73.5 & 43.1 & $<.0001$ \\
\hline Serum albumin $(\mathrm{g} / \mathrm{dL})$ & $3.82 \pm 0.78$ & $4.27 \pm 0.96$ & $<.0001$ \\
\hline hs-CRP (mg/L) & $4.82 \pm 21.42(n=70)$ & $2.64 \pm 6.82(n=311)$ & .1359 \\
\hline
\end{tabular}

Abbreviations: ACEi, angiotensin-converting enzyme inhibitor; ARB, angiotensin receptor blocker; DBP, diastolic blood pressure; hs-CRP, highsensitive C-reactive protein; iGFR, iohexol-based glomerular filtration rate; LVMI, left ventricular mass index; PP, pulse pressure; PPi, pulse pressure index; SBP, systolic blood pressure.

the dependent variable, and DBP, PP, and serum albumin as independent variables showed that DBP, PP, and serum albumin were all significantly associated with an OR of $-0.10(-0.16,-0.04 ; P=.002)$, $-0.17(-0.24,-0.11 ; P<.001)$ and $3.95(2.16,5.74 ; P<.001)$, respectively. Lastly, logistic regression analysis with $\mathrm{SCr}$ as the dependent variable, and DBP, PP, and serum albumin as independent variables was performed. DBP, PP and serum albumin were all significantly associated with an OR of $0.03(0.02,0.03 ; P<.001), 0.03(0.03,0.04$; $P<.001)$, and $-0.25(-0.38,-0.12 ; P<.001)$, respectively.

Ambulatory arterial stiffness index data were evaluated for correlations with SBP, DBP, PP, PPi, iGFR, and LVMI. The data are only presented for the overall CKD population as there was no statistically significant difference between the immunological and non-immunological group (Table 4). When analyzing the correlation for all six variables with $\mathrm{AASI}$, only $\mathrm{DBP}\left(R^{2}=.001 ; P<.001\right), \mathrm{PP}\left(R^{2}=.024\right.$; $P<.001)$, and $\mathrm{PPi}\left(R^{2}=.021 ; P<.001\right)$ showed significant correlation with AASI. AASI did not correlate with iGFR $\left(R^{2}=.004 ; P<.001\right)$, $\operatorname{SBP}\left(R^{2}=.001 ; P<.001\right)$, or LVMI $\left(R^{2}=.002 ; P<.001\right)$

\section{4 | DISCUSSION}

Assessment of vascular compliance and function in pediatric CKD populations is integral to the identification and stratification of CVD risk factors necessary for further development of treatment and preventative care strategies aimed at ameliorating long-term morbidity and mortality. Currently, arterial assessments can utilize multiple modalities including Doppler ultrasound, applanation tonometry, oscillometer, and magnetic resonance imaging. However, these modalities are often difficult to incorporate in clinical practice due to the need for specialized equipment. ${ }^{19,20}$ Furthermore, interval references for each of these measurements by sex and/ or body size have not been well defined in the pediatric population. This is the first study to provide analysis of markers of arterial compliance, including PP, PPi, and AASI, in a pediatric CKD cohort.
The authors identified an inverse relationship between increased SBP, DBP, and PP with GFR and LVMI, similar to adult CKD cohorts. The study also evaluates these factors based on CKD etiology as immunological vs non-immunological. Investigations to clarify which modality or markers are best for CVD risk stratification may perhaps be best carried out in a cohort of CKD children who have developed "hard outcomes," such as ESRD; thus, our findings are hypothesis-generating and can serve as an informative preliminary arbiter of risk discrimination.

TABLE 4 Pearson's correlation analysis

\begin{tabular}{|c|c|c|c|c|c|c|}
\hline & \multicolumn{2}{|l|}{ iGFR } & \multicolumn{2}{|l|}{$\mathrm{SCr}$} & \multicolumn{2}{|c|}{ LVMI } \\
\hline & $R^{2}$ & $P$ & $R^{2}$ & $P$ & $R^{2}$ & $P$ \\
\hline \multicolumn{7}{|c|}{ All patients } \\
\hline SBP & .033 & $<.001$ & .136 & $<.001$ & .013 & $<.001$ \\
\hline DBP & .013 & $<.001$ & .061 & $<.001$ & .005 & $<.035$ \\
\hline PP & .017 & .011 & .005 & .029 & .017 & .011 \\
\hline PPi & .001 & .309 & .001 & .265 & .001 & .437 \\
\hline AASI & .004 & $<.001$ & - & - & .002 & $<.001$ \\
\hline \multicolumn{7}{|c|}{ Immunological CKD } \\
\hline SBP & .058 & $<.001$ & .150 & $<.001$ & .104 & $<.001$ \\
\hline DBP & .070 & $<.001$ & .103 & $<.001$ & .040 & .006 \\
\hline PP & .000 & .755 & .021 & .001 & .031 & .016 \\
\hline $\mathrm{PPi}$ & .021 & .011 & .009 & .033 & .003 & .492 \\
\hline \multicolumn{7}{|c|}{ Non-immunological CKD } \\
\hline SBP & .026 & $<.001$ & .130 & $<.001$ & .003 & .156 \\
\hline DBP & .004 & .030 & .049 & $<.001$ & .001 & .512 \\
\hline PP & .017 & $<.001$ & .044 & $<.001$ & .001 & .302 \\
\hline PPi & .005 & .018 & .002 & .065 & .001 & .442 \\
\hline
\end{tabular}

Abbreviations: DBP, diastolic blood pressure; iGFR, iohexol-based glomerular filtration rate; LVMI, left ventricular mass index; PP, pulse pressure; $\mathrm{PPi}$, pulse pressure index; $\mathrm{SBP}$, systolic blood pressure; $\mathrm{SCr}$, serum creatinine. 


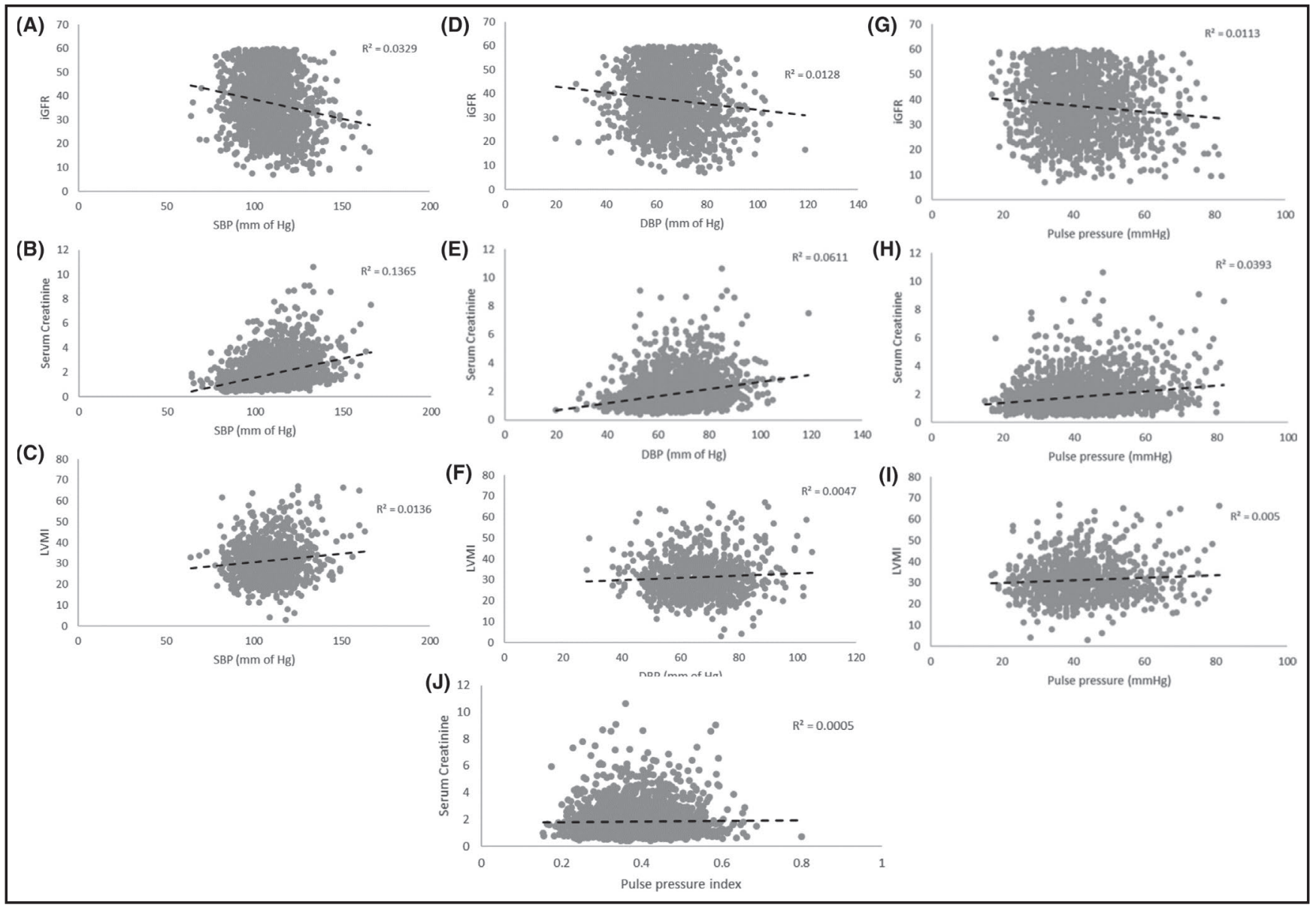

FIGURE 1 Significant correlations within the overall CKD group. SBP association with iGFR (A), SCr (B), and LVMI (C). DBP association with iGFR (D), SCr (E), and LVMI (F). PP correlation with iGFR (G), SCr (H), and LVMI (I). PPi correlation with SCr (J). iGFR, iohexol glomerular filtration rate; LMVI, left ventricular mass index; SBP, systolic blood pressure

TABLE 5 Proteinuria analysis vs pressure variables

\begin{tabular}{|c|c|c|c|c|}
\hline & $\begin{array}{l}<90 \text { th } \\
\text { percentile }\end{array}$ & $\begin{array}{l}\text { 90th } \\
\text { to 95th } \\
\text { percentile }\end{array}$ & $\begin{array}{l}>95 \text { th } \\
\text { percentile }\end{array}$ & $P$ value \\
\hline \multicolumn{5}{|c|}{ Proteinuria vs SBP } \\
\hline $\begin{array}{l}\text { Significant } \\
\text { (Up/c 0.2-2) }\end{array}$ & $60 \%$ & $67 \%$ & $60 \%$ & .11 \\
\hline $\begin{array}{l}\text { Nephrotic } \\
(\text { Up/c > 2) }\end{array}$ & $13 \%$ & $17 \%$ & $17 \%$ & .06 \\
\hline \multicolumn{5}{|c|}{ Proteinuria vs DBP } \\
\hline $\begin{array}{l}\text { Significant } \\
\text { (Up/c 0.2-2) }\end{array}$ & $62 \%$ & $67 \%$ & $54 \%$ & .38 \\
\hline $\begin{array}{l}\text { Nephrotic } \\
(\text { Up/c > 2) }\end{array}$ & $2 \%$ & $13 \%$ & $23 \%$ & .03 \\
\hline
\end{tabular}

Note: The $P$ value evaluated the linear trends across the three blood pressure levels and were compared to the normal Up/c range of $<.2$. Abbreviations: DBP, diastolic blood pressure; SBP, systolic blood pressure; Up/c, urine protein to creatinine ratio.

Pulse pressure is mainly determined by the direct force of ventricular ejection and the viscoelasticity of large arteries, and indirectly by wave reflections. ${ }^{21}$ Wave reflection is formed when forward-moving blood is partially reflected back from areas with transition in arterial impedance, for example, arterial bifurcations, stenoses, and peripheral beds. Increased arterial stiffness causes the vessel's wave reflection to become larger and arrive earlier in systole, thus greatly increasing PP. ${ }^{3}$ Endothelial stress and arteriosclerosis increase PP as loss of vascular compliance increases SBP while simultaneously decreasing DBP. ${ }^{3}$ Arteriosclerosis in CKD and ESRD patients increases arterial stiffness driving hypertension and increases PP. Elevated PP has previously been associated with adverse cardiovascular outcomes, death, and dialysis in adult patients with CKD stages 4 and $5 .{ }^{22}$ Our study is the first to investigate this relationship in a pediatric population and demonstrate a significant correlation between increased PP and decreased kidney function in all three groups of pediatric CKD patients (Figure 1).

Pulse pressure index is significantly associated with atherosclerosis, CV morbidity, and CKD in adults; these are likely due to PPi's negative correlation with vascular compliance. ${ }^{20,23,24} \mathrm{PPi}$ is also associated with increased left ventricular filling pressure and left ventricular diastolic dysfunction, which are common in adult CKD patients, especially those in pre-dialysis. ${ }^{7}$ In our overall pediatric CKD group, the authors found that PP had significant associations with iGFR, SCr, and LVMI; however, PPi was not significantly 
associated with any of the variables. Among the immunological CKD group, SCr significantly associated with both PP and PPi, iGFR correlated with PPi, and LVMI correlated only with PP. Among the non-immunological group, PP and PPi were significantly correlated with both iGFR and SCr but not LVMI. Additionally, AASI only significantly associated with PP, PPi, and DBP. The mechanism behind the variability of these associations is unclear; however, the finding that SBP and DBP always correlate with iGFR and SCr while PP and PPi do so only occasionally may be indicative of the overall vascular compliance of our young population. The loss of vascular compliance is a common end point of CKD and ESRD; however, the amount of time a patient has suffered from advanced CKD, as well as the presence of inflammation due to their disease, may be an important prognostic indicator for loss of vascular compliance in children. Additionally, proteinuria is also strongly associated with CKD progression and cardiovascular outcomes in adults. In our study, nephrotic-range proteinuria with $U p / c \geq 2$ was significantly associated with hypertensive range DBP. In regard to SBP, there was a trend toward an association with proteinuria, though it was non-significant. In general, proteinuria has been shown to accompany various types of hypertensive renal diseases in pediatric patients, such as glomerulonephritis.
Ambulatory arterial stiffness index has been established as an effective predictor of arterial stiffness in the general adult population as well as CKD patients; however, AASI has not been evaluated in a pediatric CKD population. Among adult hemodialysis patients, those with increased arterial stiffness have significantly increased risk of all-cause and cardiovascular mortality, and increased AASI also associates with early signs of renal damage in adults with primary hypertension. ${ }^{25}$ In a retrospective analysis of 418 untreated hypertensive patients, PP was identified as a crucial parameter for arterial stiffness and strong independent predictor of increasing AASI. $^{26}$ The authors found a significant association of AASI with three of our six variables of interest including PP, PPi, and DBP. The association of AASI with established markers of vascular compliance such as PP was not unexpected. However, the authors found that AASI correlated with DBP but not SBP. This is consistent with findings by Franklin et al that predictors of CVD gradually shift from DBP to SBP and then to PP with increasing age; specifically, in patients $<50$ years of age, DBP was the strongest predictor of CVD. ${ }^{21}$ This gradual age-based change is the result of how cardiac afterload is affected by arterial stiffening. Peak SBP in the ascending aorta is affected by peripheral SBP distortion secondary to wave reflection in older individuals. In young hypertensives

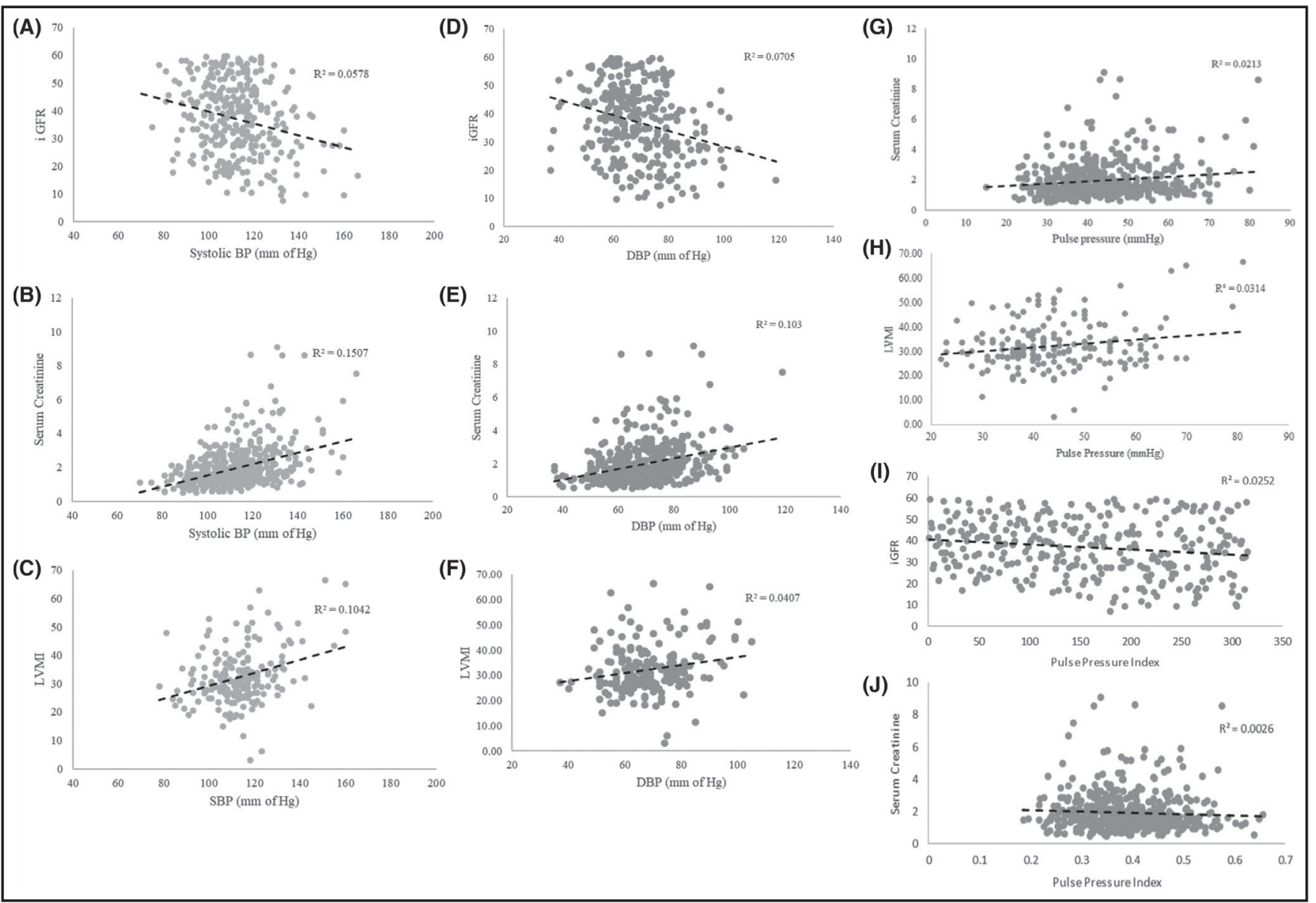

FIGURE 2 Significant correlation within the immunological CKD group. SBP association with iGFR (A), SCr (B), and LVMI (C). DBP association with iGFR (D), SCr (E), and LVMI (F). PP correlation with $\mathrm{SCr}(\mathrm{G})$, and LVMI (H). PPi correlation with iGFR (I) and SCr (J). iGFR, iohexol glomerular filtration rate; LMVI, left ventricular mass index; SBP, systolic blood pressure 
TAB LE 6 Backward logistic regression analysis for immunological vs non-immunological CKD

\begin{tabular}{|c|c|c|c|c|}
\hline & $\begin{array}{l}\text { Odds } \\
\text { Ratio }\end{array}$ & $\begin{array}{l}95 \% \mathrm{Cl} \\
\text { High }\end{array}$ & $\begin{array}{l}95 \% \mathrm{Cl} \\
\text { Low }\end{array}$ & $P$ value \\
\hline \multicolumn{5}{|l|}{ Immunological CKD } \\
\hline \multicolumn{5}{|l|}{ LVMI } \\
\hline DBP & 0.16 & 0.28 & 0.05 & .005 \\
\hline PP & 0.20 & 0.32 & 0.07 & .002 \\
\hline Serum albumin & -4.84 & -1.78 & -7.89 & .002 \\
\hline \multicolumn{5}{|l|}{ iGFR } \\
\hline DBP & 0.27 & -0.16 & -0.38 & $<.001$ \\
\hline \multicolumn{5}{|l|}{$\mathrm{SCr}$} \\
\hline DBP & 0.03 & 0.04 & 0.03 & $<.001$ \\
\hline PP & 0.02 & 0.03 & 0.01 & $<.001$ \\
\hline \multicolumn{5}{|c|}{ Non-Immunological CKD } \\
\hline \multicolumn{5}{|l|}{ LVMI } \\
\hline SBP & -2.63 & -0.86 & -4.41 & .004 \\
\hline \multicolumn{5}{|l|}{ iGFR } \\
\hline DBP & -0.10 & -0.04 & -0.16 & .002 \\
\hline PP & -0.17 & -0.11 & -0.24 & $<.001$ \\
\hline Serum albumin & 3.95 & 5.74 & 2.16 & $<.001$ \\
\hline \multicolumn{5}{|l|}{$\mathrm{SCr}$} \\
\hline DBP & 0.03 & 0.03 & 0.02 & $<.001$ \\
\hline PP & 0.03 & 0.04 & 0.03 & $<.001$ \\
\hline Serum albumin & -0.25 & -0.12 & -0.38 & $<.001$ \\
\hline
\end{tabular}

Abbreviations: DBP, diastolic blood pressure; iGFR, iohexol-based glomerular filtration rate; LVMI, left ventricular mass index; PP, pulse pressure; $\mathrm{SCr}$, serum creatinine.

patients, reduced peripheral amplification of SBP due to altered wave reflection leads a greater peripheral increase in DBP than in SBP while there is a parallel rise in both central SBP and DBP. Thus, in young individuals, elevated peripheral DBP is superior in predicting CVD. ${ }^{21,27}$ However, this finding will need to be replicated in a prospectively analyzed pediatric CKD population, and more observations are necessary in order to truly elucidate AASI's associations with CVD and long-term cardiovascular morbidity in our specific population.

$\mathrm{LVH}$ is the strongest risk factor for CVD in hypertensive populations. LVH is common among adult CKD patients and also occurs in $17 \%-49 \%$ of children with CKD. ${ }^{28,29}$ Reduction of LVM correlates with decreased CV events and mortality in adult hypertensive patients as well as in CKD stage 5 patients on renal replacement therapy. ${ }^{28,29}$ Common risk factors for LVH in CKD include anemia, fluid overload, and hypertension. ${ }^{28}$ In our cohort, SBP and DBP significantly correlated with LVMI in the overall CKD group and immunological CKD group. However, LVMI did not correlate with SBP nor $\mathrm{DBP}$ in the non-immunological group. This finding is consistent with adult CKD data that immunological markers such as CRP, interleukin-6 (IL-6), and tumor necrosis factor-alpha (TNF-alpha) are LVH risk factors. ${ }^{30}$ Furthermore, inflammation is also highly prevalent in pre-dialysis CKD patients and immunological markers are associated with increased LVMI in adult CKD patients. ${ }^{31,32}$

Studies have established the association of low-grade chronic inflammation and immunological markers with progression of CKD and all-cause mortality., ${ }^{9,12}$ The underlying mechanisms of chronic low-grade inflammation in CKD include increased production and reduced clearance of pro-immunomodulatory cytokines, oxidative stress, acidosis, chronic or recurrent infections, altered metabolism of adipose tissue, and intestinal dysbiosis. ${ }^{10,33}$ Various immunological biomarkers, including IL-1, IL-6, TNF-alpha, TNF-beta, hs-CRP, fibrinogen, and serum albumin, have been implicated in the progression of CKD. ${ }^{12}$ Furthermore, increased levels of immunological markers (fibrinogen and TNF-alpha) and reduced levels of serum albumin are associated with rapid loss of renal functions in pediatric CKD patients. ${ }^{12}$ However, the exact mechanism by which inflammation contributes to CKD progression is unknown. ${ }^{10}$ While our study did include the immunological marker hs-CRP, the authors did not find a significant difference in hs-CRP levels between patients with immunological and non-immunological CKD. However, the authors did demonstrate that serum albumin was significantly lower in the immunological than the non-immunological CKD group. Unfortunately, the relationship between hs-CRP, serum albumin, and iGFR could not be established due to variation in data collection time points.

Inflammation has been linked to elevated blood pressure and cardiovascular morbidity and mortality. ${ }^{34,35}$ Approximately $50 \%$ of CKD patients have increased mortality due to CV complications, such as advanced calcific arterial and valvular disease, which has been linked to chronic inflammation. ${ }^{36}$ Probable underlying pathogenesis includes an imbalance between vasoconstrictors and vasodilators, increased thrombogenesis, platelet activation, and the direct effect of immunological mediators. ${ }^{35}$ Increased levels of CRP, IL-6, and leukocyte adhesion molecules were shown to predict the risk of CV events. ${ }^{13}$ Our study showed significant elevations in SBP, DBP, and PP in the immunological CKD group (Figure 2) compared to the non-immunological group (Figure 3). In addition, both PP and DBP were significantly associated with LVMI on regression analysis for the immunological CKD group but not the non-immunological group. ACEi/ARBs were significantly higher among the immunological CKD group implying higher blood pressures among these patients (Table 3 ). In the future, prospective studies should be conducted in order to further corroborate the results shown.

\section{5 | CONCLUSIONS}

This study is one of the first to assess a pediatric CKD cohort using noninvasive surrogate markers of arterial stiffness. The authors have demonstrated an inverse relationship between SBP, DBP, and PP with decreased GFR and elevated LVMI in children, similar to adults. In addition, the authors have demonstrated differences in these relationships by CKD etiology as immunological or non-immunological, which is unique and hypothesis-generating. Our immunological CKD 

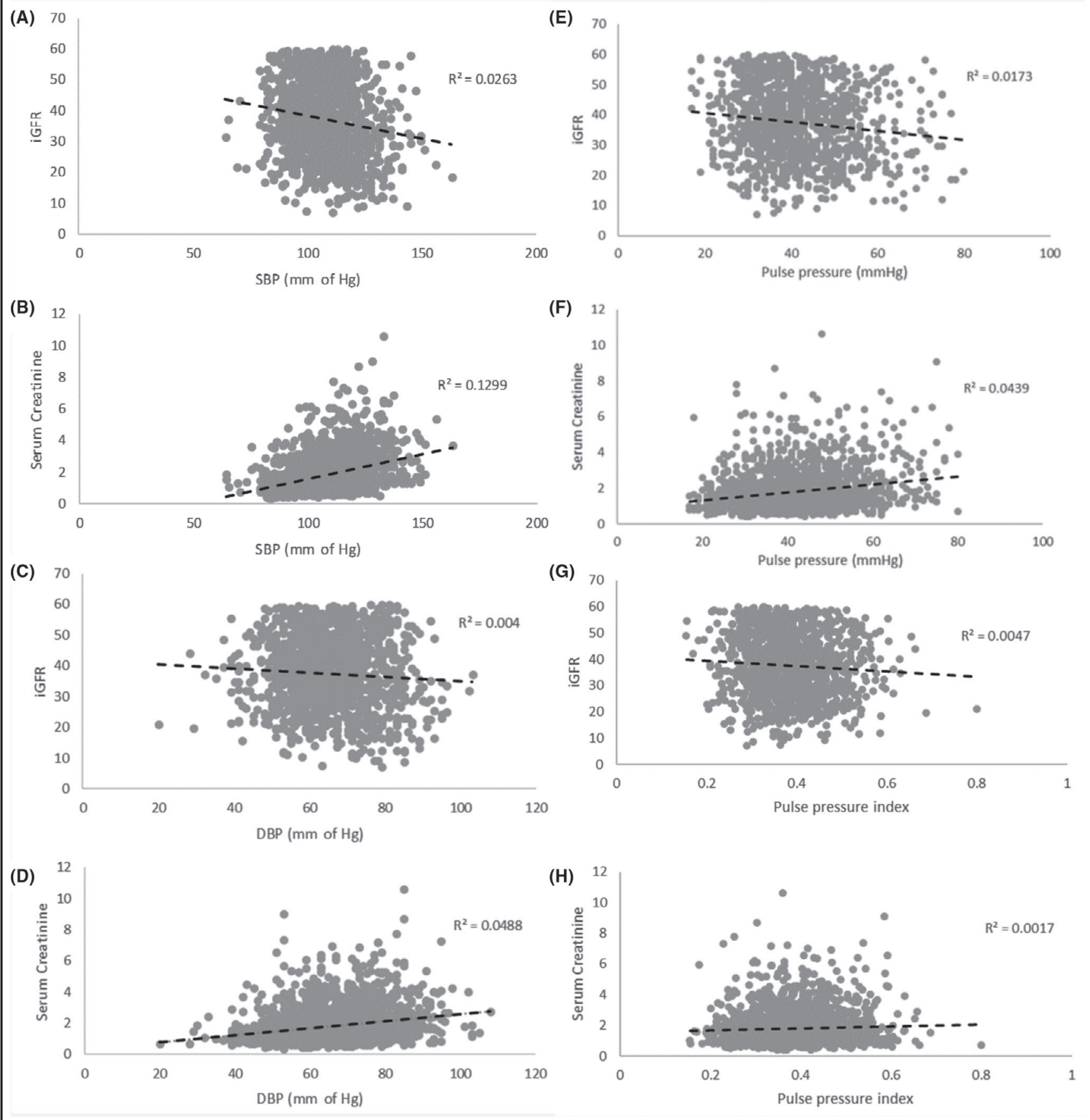

FIGURE 3 Significant correlation within the non-immunological CKD group. SBP association with iGFR (A) and SCr (B). DBP association with iGFR (C) and SCr (D). PP correlation with iGFR (E) and SCr (F). PPi correlation with iGFR (G) and SCr (H). iGFR, iohexol glomerular filtration rate; LMVI, left ventricular mass index; SBP, systolic blood pressure

subgroup children showed significantly higher SCr, SBP, DBP, PP and significantly lower serum albumin levels. Subgroup analysis of CKD patients demonstrated that SBP, DBP, and PP were all significantly correlated with LVMI in immunological CKD patients but not nonimmunological. These findings suggest that effective blood pressure control is of paramount importance in children with CKD due to immunological causes to decrease their long-term CV morbidity.

\section{ACKNOWLEDGMENTS}

The data and samples from the CKiD Study reported here were supplied by the National Institute of Diabetes and Digestive and Kidney Diseases (NIDDK) Central Repositories. This manuscript does not necessarily reflect the opinions or views of the CKiD Study, the NIDDK Central Repositories, or the NIDDK. 


\section{CONFLICT OF INTEREST}

None.

\section{AUTHOR CONTRIBUTIONS}

RR, SP, NN, RC, SS, VK, GK, MM, and KK contributed to the conception and design of this study, were involved in the data analysis and interpretation of the data, drafted the article and revised it critically for important intellectual content, and have approved the final version of this manuscript for submission.

\section{ORCID}

Rupesh Raina iD https://orcid.org/0000-0003-3892-8376 Ronith Chakraborty (iD https://orcid.org/0000-0003-1865-9682 Sidharth Sethi (iD https://orcid.org/0000-0002-1516-3393 Vinod Krishnappa iD https://orcid.org/0000-0002-1697-4526

Kirsten Kusumi iD https://orcid.org/0000-0003-3099-2226

\section{REFERENCES}

1. Arulkumaran N, Diwakar R, Tahir Z, Mohamed M, Kaski JC, Banerjee D. Pulse pressure and progression of chronic kidney disease. J Nephrol. 2010;23(2):189-193.

2. László A, Reusz GS, Nemcsik J. Ambulatory arterial stiffness in chronic kidney disease: a methodological review. Hypertens Res. 2016;39(4):192-198.

3. Fernandez-Fresnedo G, Rodrigo E, de Francisco AL, de Castro SS, Castaneda O, Arias M. Role of pulse pressure on cardiovascular risk in chronic kidney disease patients. J Am Soc Nephrol. 2006;17(12 Suppl 3):S246-S249.

4. Nichols WM. Clinical measurement of arterial stiffness obtained from noninvasive pressure waveforms. Am J Hypertens. 2005;18(1 Pt 2):3S-10S.

5. Matsui Y, Kario K, Ishikawa J, Eguchi K, Hoshide S, Shimada K. Reproducibility of arterial stiffness indices (pulse wave velocity and augmentation index) simultaneously assessed by automated pulse wave analysis and their associated risk factors in essential hypertensive patients. Hypertens Res. 2004;27(11):851-857.

6. Peng-Lin Y, Yue-Chun L. Pulse pressure index (pulse pressure/systolic pressure) may be better than pulse pressure for assessment of cardiovascular outcomes. Med Hypotheses. 2009;72(6):729-731.

7. Li Y, Wang J, Dolan E, et al. Ambulatory arterial stiffness index derived from 24-hour ambulatory blood pressure monitoring. Hypertension. 2006;47:359-364.

8. Lee WH, Hsu PC, Chu CY, et al. Associations of pulse pressure index with left ventricular filling pressure and diastolic dysfunction in patients with chronic kidney disease. Am J Hypertens. 2014;27(3):454-459.

9. Bansal N, Lin F, Vittinghoff E, et al. Estimated GFR and subsequent higher left ventricular mass in young and middle-aged adults with normal kidney function: the coronary artery risk development in young adults (CARDIA) study. Am J Kidney Dis. 2016;67(2):227-234.

10. Gupta J, Mitra N, Kanetsky PA, et al. Association between albuminuria, kidney function, and inflammatory biomarker profile in CKD in CRIC. Clin J Am Soc Nephrol. 2012;7(12):1938-1946.

11. Akchurin OM, Kaskel F. Update on inflammation in chronic kidney disease. Blood Purif. 2015;39(1-3):84-92.

12. Goldstein SL, Leung JC, Silverstein DM. Pro- and anti-inflammatory cytokines in chronic pediatric dialysis patients: effect of aspirin. Clin J Am Soc Nephrol. 2006;1(5):979-986.
13. Amdur RL, Feldman HI, Gupta J, et al. Inflammation and progression of CKD: the CRIC study. Clin J Am Soc Nephrol. 2016;11(9):1546-1556.

14. Ridker PM, Hennekens CH, Buring JE, Rifai N. C-reactive protein and other markers of inflammation in the prediction of cardiovascular disease in women. N Engl J Med. 2000;342(12):836-843.

15. Samuels J, Ng D, Flynn JT, et al. Ambulatory blood pressure patterns in children with chronic kidney disease. Hypertension. 2012;60(1):43-50

16. Mitsnefes M, Flynn J, Cohn S, et al. Masked hypertension associates with left ventricular hypertrophy in children with CKD. J Am Soc Nephrol. 2010;21(1):137-144.

17. Borzych D, Bakkaloglu SA, Zaritsky J, et al. Defining left ventricular hypertrophy in children on peritoneal dialysis. Clin J Am Soc Nephrol. 2011;6(8):1934-1943.

18. Fuhrman DY, Schneider MF, Dell KM, et al. Albuminuria, proteinuria, and renal disease progression in children with CKD. Clin J Am Soc Nephrol. 2017;12(6):912-920.

19. Ku E, McCulloch CE, Warady BA, Furth SL, Grimes BA, Mitsnefes MM. Twenty-four-hour ambulatory blood pressure versus clinic blood pressure measurements and risk of adverse outcomes in children with CKD. Clin J Am Soc Nephrol. 2018;13(3):422-428.

20. Cai A, Mo Y, Zhang $Y$, et al. Relationship of pulse pressure index and carotid intima-media thickness in hypertensive adults. Clin Exp Hypertens. 2015;37(4):267-270.

21. Franklin SS, Larson MG, Khan SA, et al. Does the relation of blood pressure to coronary heart disease risk change with aging? The Framingham Heart Study. Circulation. 2001;103(9):1245-1249.

22. Lee MK, Hsu PC, Chu CY, et al. Significant correlation between Brachial Pulse Pressure Index and Renal Resistive Index. Acta Cardiol Sin. 2015;31(2):98-105.

23. Rahman M, Hsu JY, Desai N, et al. Central blood pressure and cardiovascular outcomes in chronic kidney disease. Clin J Am Soc Nephrol. 2018;13(4):585-595.

24. Zhao YJ, Shen LH, Wang W, et al. Clinical value of pulse pressure, pulse pressure index and estimated glomerular filtration rate in patients with essential hypertension. J Shanghai Jiaotong Univ (Medical Science). 2007;27:1258-1260.

25. Kwarciany M, Gasecki D, Kowalczyk K, et al. Acute hypertensive response in ischemic stroke is associated with increased aortic stiffness. Atherosclerosis. 2016;251:1-5.

26. Lee HT, Lim YH, Kim BK, et al. The relationship between ambulatory arterial stiffness index and blood pressure variability in hypertensive patients. Korean Circ J. 2011;41(5):235-240.

27. Dolan E, Thijis L, Li Y, et al. Ambulatory arterial stiffness index as a predictor of cardiovascular mortality in the Dublin Outcome Study. Hypertension. 2006;47(3):365-370.

28. Port S, Demer L, Jennrich R, Walter D, Garfinkel A. Systolic blood pressure and mortality. Lancet. 2000;355(9199):175-180.

29. Kupferman JC, Aronson Friedman L, Cox C, et al. BP control and left ventricular hypertrophy regression in children with CKD. J Am Soc Nephrol. 2014;25(1):167-174

30. Ioannou K, Stel VS, Dounousi E, et al. Endothelial dysfunction and increased left ventricular mass in chronic kidney disease (CKD) patients: a longitudinal study. PLoS One. 2015;10(9):e0138461.

31. Zoccali C, Benedetto FA, Mallamaci F, et al. Left ventricular mass monitoring in the follow-up of dialysis patients: prognostic value of left ventricular hypertrophy progression. Kidney Int. 2004;65(4):1492-1498.

32. Cottone S, Nardi E, Mule G, et al. Association between biomarkers of inflammation and left ventricular hypertrophy in moderate chronic kidney disease. Clin Nephrol. 2007;67(4):209-216.

33. Dervisoglu E, Kozdag G, Etiler N, Kalender B. Association of glomerular filtration rate and inflammation with left ventricular 
hypertrophy in chronic kidney disease patients. Hippokratia. 2012;16(2):137-142.

34. Mihai S, Codrici E, Popescu ID, et al. Inflammation-related mechanisms in chronic kidney disease prediction, progression, and outcome. J Immunol Res. 2018;2018:1-16.

35. Subasinghe AK, Wark JD, Gorelik A, Callegari ET, Garland SM. The association between inflammation, obesity and elevated blood pressure in 16-25-year-old females. J Hum Hypertens. 2017;31(9):580-584.

36. Ghanem FA, Movahed A. Inflammation in high blood pressure: a clinician perspective. J Am Soc Hypertens. 2007;1(2):113-119.
How to cite this article: RainaR, Polaconda S, Nair N, et al. Association of pulse pressure, pulse pressure index, and ambulatory arterial stiffness index with kidney function in a cross-sectional pediatric chronic kidney disease cohort from the CKiD study. J Clin Hypertens. 2020;22:1059-1069. https://doi.org/10.1111/jch.13905 